This document is the accepted manuscript version of the following article:

Gavriilidou, A. F. M., Holding, F. P., Mayer, D., Coyle, J. E., Veprintsev, D. B., \& Zenobi, R. (2018). Native mass spectrometry gives insight into the allosteric binding mechanism of M2 pyruvate kinase to fructose-1,6-bisphosphate. Biochemistry, 57(11), 1685-1689. https://doi.org/10.1021/acs.biochem.7b01270

\title{
Native mass spectrometry gives insight into the allosteric binding mechanism of M2 pyruvate kinase to fructose-1,6- bisphosphate
}

\author{
Agni F. M. Gavriilidou ${ }^{1}$, Finn P. Holding ${ }^{2}$, Daniel Mayer ${ }^{3}$, Joseph E. Coyle ${ }^{2}$, Dmitry B. Veprintsev ${ }^{3 \ddagger}$ \\ Renato Zenobi ${ }^{1 *}$ \\ ${ }^{1}$ ETH Zurich, Department of Chemistry and Applied Biosciences, CH-8093 Zurich, Switzerland \\ ${ }^{2}$ Astex Pharmaceuticals, 436 Cambridge Science Park, Milton Road, Cambridge, CB4 0QA, United Kingdom \\ ${ }^{3}$ Paul Scherrer Institute, 5232 Villigen PSI (Switzerland) \\ †Current address: Centre of Membrane Proteins and Receptors, School of Life Sciences, University of Nottingham, NG7 \\ 2UH, UK
}

\begin{abstract}
The various oligomeric states of the M2 isoform of pyruvate kinase (PKM2) were distinguished using native mass spectrometry (MS). The effect of PKM2 concentration on its dimer:tetramer equilibrium was monitored and a value for the dissociation constant $\left(\mathrm{K}_{\mathrm{d}}\right)$ of the two species was estimated to be $0.95 \mu \mathrm{M}$. Results of binding experiments of fructose-1,6bisphosphate (FBP) to PKM2 are shown, which provide insight into the allosteric mechanism and changes in the oligomerization status of PKM2. The average $\mathrm{K}_{\mathrm{d}}$ of FBP to PKM2 tetramer was estimated to be $7.5 \mu \mathrm{M}$. It is concluded that four molecules of FBP bind to the active PKM2 tetramer whereas binding of FBP to the PKM2 dimer was not observed. It is suggested that either FBP potentiates rapid tetramer formation after binding to apo PKM2 dimers or FBP binds to PKM2 apo tetramers thus driving the dimer:tetramer equilibrium in the direction of fully FBP bound tetramer. The binding occurs in a highly positively cooperative manner with a Hill coefficient of $n=3$.
\end{abstract}

Pyruvate kinase $(\mathrm{PK})$ is one of three regulatory enzymes in the glycolytic pathway and catalyzes the last step by transferring a phosphate group from phosphoenolpyruvate (PEP) to adenosine di-phosphate (ADP), yielding one molecule of pyruvate and one molecule of adenosine tri-phosphate (ATP) ${ }^{1}$. Four isoforms of PK are expressed in mammals: PKL, PKR, PKM1 and PKM2, and cancer cells have been shown to preferentially express the M2 isoform ${ }^{2}$. Kinetic characterization of the tetrameric and dimeric form of PKM2 has revealed that the tetrameric form has a high affinity for PEP resulting in increased ATP production, whereas the dimeric form has low affinity for PEP and reduced ATP production $^{2}$. In tumor cells, the inactive, dimeric, form of PKM2 predominates and glycolytic metabolites are thus diverted to anabolic pathways in order to maintain tumor growth $^{3,4}$. Extensive biochemical characterization has previously been undertaken to investigate the pyruvate kinase activity of PKM2 over other PK isoforms in the presence of the native allosteric activator FBP and other small molecule ligands, and how FBP binding regulates the activity of PKM2 and glucose uptake during glycolysis in tumor and non-tumor cells ${ }^{5}$. Various compounds have been tested for their ability to activate PKM2 and suppress tumor proliferation ${ }^{6,7}$. Molecular dynamic simulations were applied to study the atomic relationship between FBP and serine for allosteric regulation of PKM2 $2^{8}$. PKM2 has been shown to be critical for cancer metabolism and tumor growth compared to PKM1 due to diminished tumor proliferation when PKM2 was replaced by its isoform PKM1 $1^{5,9}$. PKM1 has been studied by electrospray ionization mass spectrometry coupled to hydrogen-deuterium exchange to analyze the mechanisms of activation and allosteric regulation ${ }^{10}$. Distinguishing the various active and inactive species of PKM2 has obvious benefit in the design and testing of novel therapeutic agents. However, the allosteric mechanism by which FBP binds to and activates PKM2, and its effect on the dimer/tetramer ratio of the protein, remains poorly understood.

Native electrospray ionization mass spectrometry (ESI-MS) is a rapid, sensitive and high resolution approach which has previously been utilized to analyze allosteric mechanisms ${ }^{11,12}$. It can determine composition, stoichiometry, subunit interactions, binding affinity and architectural organization of noncovalent protein complexes ${ }^{13-16}$. Native MS has been utilized to study apo pyruvate kinase ${ }^{17,18}$ and in the current study native MS was applied to provide insights into the allosteric regulation and cooperativity of FBP binding to PKM2. The oligomeric states of PKM2 were analyzed in the absence and presence of FBP and values for the stoichiometry of FBP binding and the affinity were determined by FBP titration experiments. The Hill coefficient was also calculated by fitting the data to the Hill equation in order to determine cooperativity of FBP binding to the four PKM2 monomers. The S437Y PKM2 mutant which contains a sterically occluded FBP binding site was used as a negative control.

The equilibrium between dimers and tetramers is dependent on the presence of multiple regulatory molecules ${ }^{8}$ including serine, $\mathrm{K}^{+}$and $\mathrm{NH}_{4}^{+}$ions and $\mathrm{FBP}$, as well as the protein concentration $^{5,7}$ (Figure 1). In the absence of FBP PKM2 was found by $\mathrm{MS}$ to be predominantly dimeric $(86.0 \% \pm 7.6$ at $2.5 \mu \mathrm{M}$ dimeric PKM2, mean of 6 replicates), an observation which was also made previously by isoelectric focusing chromatography ${ }^{3}$. Morgan et al. ${ }^{19}$ and Anastasiou et. al. ${ }^{5}$ observed monomeric PKM2 by SEC-MALS and ultracentrifugation, respectively, but in our MS experiments the monomer was not found at high abundance. Native MS is a less energetic technique, i.e., dissociation of tetramers to monomers caused by the experimental conditions is expected 
to be minimal compared to other techniques. Monomer and trimer species were observed only with very low intensity (Figure 2a).

The trimer population is more prominent at high concentrations of PKM2 (Figure 1a); formation of nonspecific higher order oligomers may be driven by the electrospray process at increased protein concentration as the probability of individual electrospray droplet containing more than one analyte molecule is increased.

It is noteworthy that FBP did not affect the distribution of monomers with respect to the other oligomeric states (Figure S1). Moreover, the inactive dimeric form of PKM2 has been found in cancer cells ${ }^{2,3}$ rendering it of increased interest compared to the low abundance monomer population.

The relationship between the ion signal and the analyte concentration in an ESI mass spectrometer is referred to as the response factor ' $R$ '. It is the result of various parameters affecting the ion signal intensities including the ionization probability of the various species present in solution, the differences in ion transmission through the mass spectrometer and the MS detection efficiency ${ }^{20}$.

To correlate the relative intensities of dimers and tetramers in the mass spectra with their relative abundance in solution, the response factors of the dimer, $\mathrm{R}_{\mathrm{D}}$, and the of tetramer, $\mathrm{R}_{T}$, were taken into account during the analysis of the MS data. In order to determine the ratio of the response factors, equation (1) was used. The PKM2 concentration was increased incrementally and the equilibrium between the two oligomeric states was found to shift toward tetramer at increased protein concentrations.

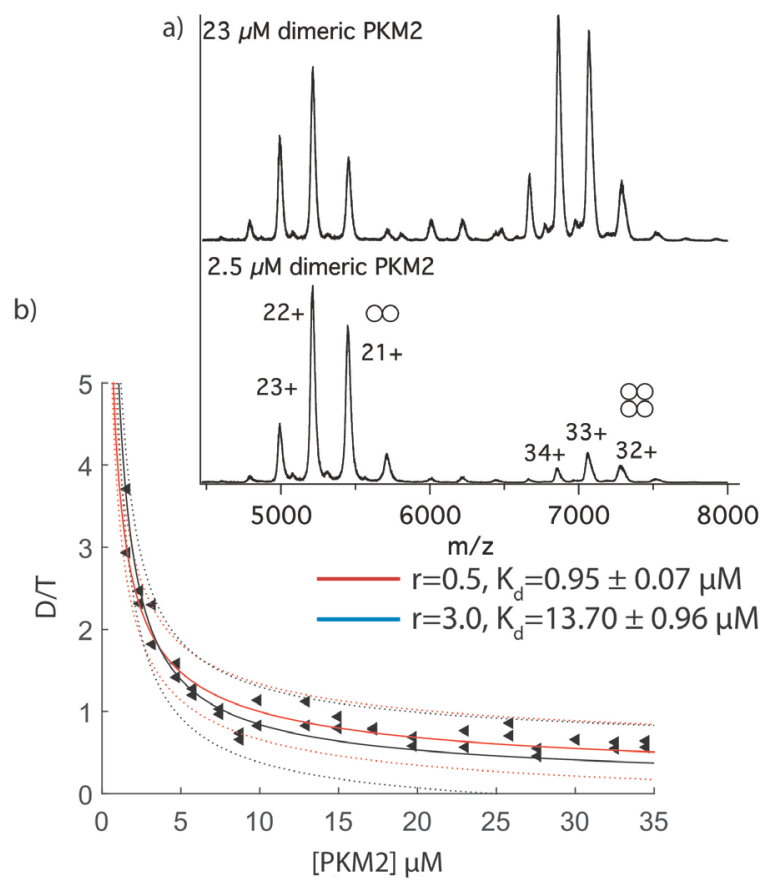

Figure 1: a) Native ESI mass spectra of wild type PKM2 at dimer concentration of $2.5 \mu \mathrm{M}$ and $23.0 \mu \mathrm{M}$. The tetramer is represented by four circles and the dimer by two. b) ) Plot of the ratio of the experimentally determined peak areas (black triangles) representing dimer (D) and tetramer (T) populations versus the dimer concentration [PKM2]. To ensure reproducibility, the data were collected on two separate days from different samples. Two curves are shown for response factor ratio $(\mathrm{r})=0.5$ (red) and $\mathrm{r}=3.0$ 2 (blue) and a 95\% confidence interval is indicated with the dotted lines for each case. The ratio of the dimer and tetramer ionization, $\mathrm{r}$, and the dissociation constant $\left(\mathrm{K}_{\mathrm{d}}\right)$ of the dimer:dimer interaction were subsequently calculated.

The curve in Figure 1 was generated by a non-linear fitting to the following equation ${ }^{21}$ :

$\frac{\mathrm{D}}{\mathrm{T}}=\frac{1+\sqrt{1+8 * \mathrm{~K}_{\mathrm{a}} * \mathrm{c}}}{2 * \mathrm{r} * \mathrm{~K}_{\mathrm{a}} * \mathrm{c}}$

$\mathrm{D}=$ peak area of the dimer, $\mathrm{T}=$ peak area of the tetramer, $\mathrm{K}_{\mathrm{a}}=$ association constant of the dimer:dimer interaction $\left(K_{a}=1 / K_{d}\right), r=$ ratio between the response factors of the dimer and the tetramer and $\mathrm{c}=$ concentration of the dimer determined by $A_{280}\left(\varepsilon=29790 \mathrm{M}^{-1} \mathrm{~cm}^{-1}\right.$ for the monomer $)$ measurement. In order to facilitate data fitting, the $r$ value was restricted to a range between 0.5 and 6 , based on literature data ${ }^{21}$. Using nonlinear fitting with a bi-square robust algorithm that minimizes the weighted sum of squares, the best fit to the data with a $95 \%$ confidence interval was achieved with $\mathrm{r}=0.5$ (Figure 1). This value was therefore applied to subsequent calculations and the dimer:dimer $\mathrm{K}_{\mathrm{d}}$ value calculated to be $0.95 \mu \mathrm{M}$. The value of $r=0.5$ suggests that the response factor of the tetramer is higher than that of the dimer. This is in agreement with the literature $^{22}$, where it has been shown that a larger protein is expected to give a stronger signal in the mass spectrum.

It has previously been suggested that FBP activates PKM2 by inducing conformational changes which promote its tetramerization ${ }^{5}$. The effect of increasing concentrations of FBP on the oligomeric states of PKM2 was tested (Figure 2a). FBP was titrated into $2.5 \mu \mathrm{M}$ PKM2 (dimer concentration, corresponding to $5.0 \mu \mathrm{M}$ monomer) and four molecules of FBP were observed to bind to the PKM2 tetramer whereas FBP was not observed binding to the dimer. The ratio of dimer/tetramer clearly decreased in the presence of increasing [FBP] suggesting that FBP induced a conformational change in the dimer that potentiated tetramer formation. It is noteworthy that even after extensive purification and desalting the protein was still observed with $11.0 \% \pm 1.4$, (6 replicates) or $0.5 \mu \mathrm{M}$ of endogenous FBP bound (lower spectrum of Figure $2 \mathrm{~b} \&$ Figure S2), which was taken into account during subsequent calculations.

At FBP concentration $=4.5 \mu \mathrm{M}+0.5 \mu \mathrm{M}$ endogenous FBP PKM2 was found to be predominantly tetrameric $(71.0 \% \pm$ 4.4, Figure 3). This result agrees with previous reports showing that the fully associated tetrameric form of PKM2 is promoted in the presence of activators ${ }^{5}$. In Figure $2 b$, zoomed spectra of the PKM2 tetramer at different FBP concentrations are shown. Before saturation of the tetrameric form is reached, two oligomer populations were distinguished, fully FBP bound $(233615 \pm 72 \mathrm{Da})$ tetramer and apo tetramer $(232146 \pm 70$ Da). The mass difference between the two populations (1496 $\mathrm{Da}$ ) clearly corresponds to four molecules of FBP (theoretical mass of FBP $=340 \mathrm{Da}$ ). The ratio of the areas of the FBP bound PKM2 peaks to the total area of all the peaks were plotted against FBP concentration (Figure 3).

Application of the Hill equation yielded a Hill coefficient of 3 which is indicative of a highly cooperative binding interaction. An apparent average binding affinity of $7.5 \mu \mathrm{M}$ was also calculated. However, in the absence of an appropriate model for binding of FBP to PKM2 it is not possible to unequivocally calculate a single value for FBP binding. This is 
due to necessary assumptions regarding FBP binding coupled to the dimer:tetramer equilibrium, the presence of endogenous FBP and the sequential positively cooperative nature of FBP binding.

Isothermal titration calorimetry (ITC) was used to determine the affinity and stoichiometry of FBP binding to PKM2 to benchmark the results from MS experiments. ITC curves were acquired in the gel filtration buffer, $25 \mathrm{mM}$ Tris base, $\mathrm{pH} 7.5$, $100 \mathrm{mM} \mathrm{KCl}, 5 \mathrm{mM} \mathrm{MgCl}$ and in the native MS buffer, 200 $\mathrm{mM}$ ammonium acetate $\mathrm{pH} 7.5\left(\mathrm{NH}_{4} \mathrm{Ac}\right)$, (Figure S3). The binding isotherm in both solutions yielded a similar biphasic shape indicating that at least two sequential binding events occurred. The shape of the curve implied positive cooperativity which is in agreement with the result from the MS experiment. Also in accordance with the MS data, it was not possible to calculate a single $K_{d}$ value due to the lack of a suitable model for the binding of FBP to PKM2. Additional studies would be required to derive the relevant binding model and to determine $K_{d}$ values for each binding event and for the dimer to tetramer interaction.
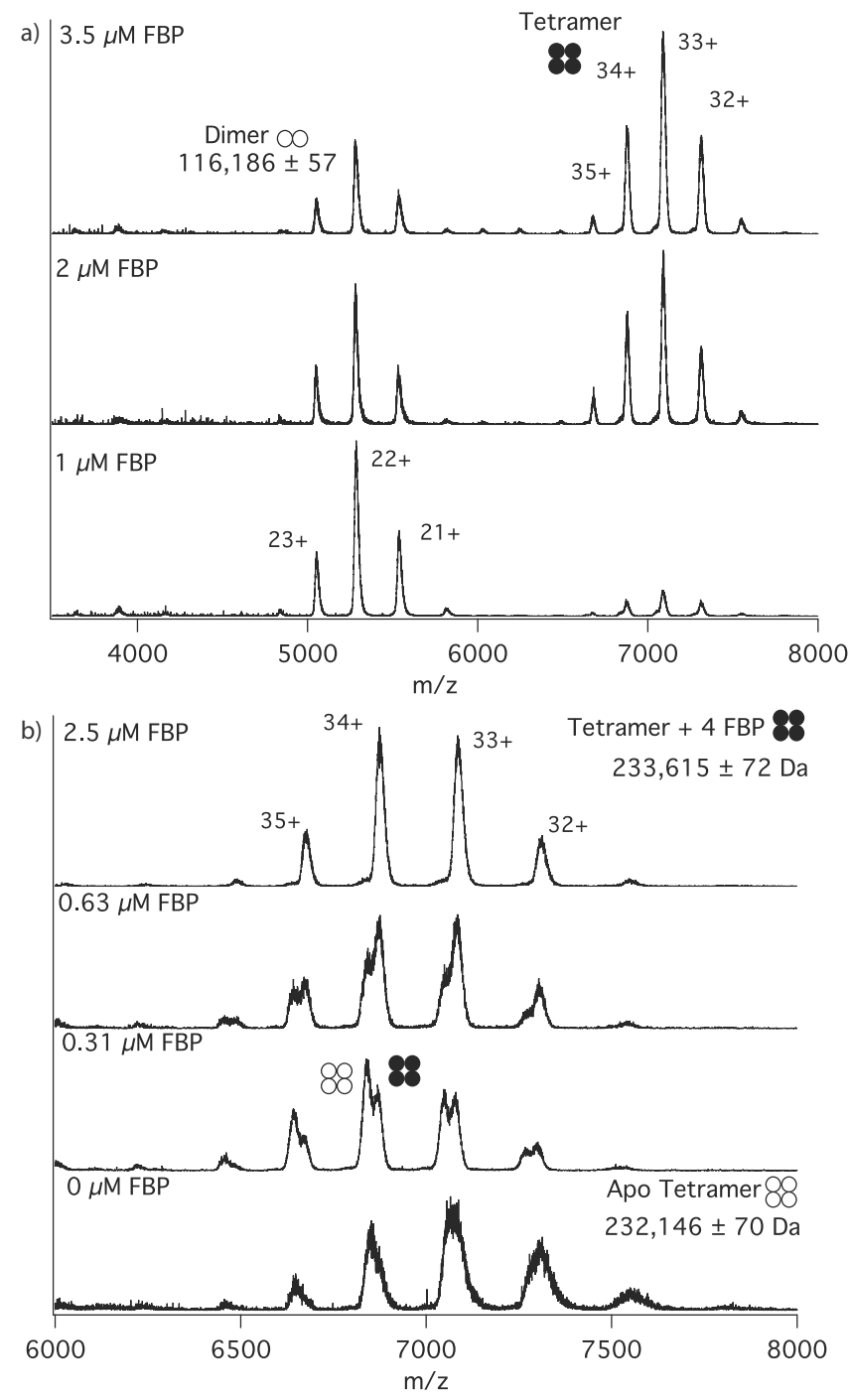

Figure 2: a) Representative nano ESI-MS spectra of $2.5 \mu \mathrm{M}$ dimeric concentration PKM2 titrated against FBP in $200 \mathrm{mM}$ NH4Ac. The intensity of the dimer (two circles) decreased and the tetramer (four circles) increased with increasing FBP concentration. b) Representative nano ESI-MS spectra of the
PKM2 tetramer (empty circles) titrated against FBP in $200 \mathrm{mM}$ NH4Ac. Four molecules of FBP are bound to the tetramer (filled circles). Before saturation was reached unbound tetramer and fully bound tetramer were observed. The mass error was derived from the standard deviation of six measurements.

Ammonium ions have also been shown previously to regulate the activity of $\mathrm{PKM} 2^{23}$ therefore the effect of ammonium ions on the oligomeric distribution of PKM2 was investigated (Figure S4). At high $\mathrm{NH}_{4} \mathrm{Ac}$ concentrations $(>1 \mathrm{M})$ the spectral intensity of the dimers series decreased whilst that of the tetramers increased. The dimer to tetramer transition in the presence of FBP occurred at micromolar concentrations, thus the relative effect of $\mathrm{NH}_{4} \mathrm{Ac}$ on the oligomeric distribution was considered to be negligible.

In order to further examine the effect of FBP on PKM2 in both Tris base and $\mathrm{NH}_{4} \mathrm{Ac}$ solutions, and to corroborate the findings from ITC and native MS, a thermal shift assay was performed to measure changes in the thermal stability of the protein (Figure S5). The $\mathrm{T}_{\mathrm{m}}$ of apo PKM2 was $47.4{ }^{\circ} \mathrm{C}$ in $\mathrm{NH}_{4} \mathrm{Ac}$ and $48.4{ }^{\circ} \mathrm{C}$ in Tris buffer, which indicated that PKM2 stability is minimally affected by the nature of the two buffers tested.

Addition of $200 \mu \mathrm{M}$ FBP to $60 \mu \mathrm{M}$ PKM2 increased the $\mathrm{T}_{\mathrm{m}}$ to $64{ }^{\circ} \mathrm{C}$ in $\mathrm{NH}_{4} \mathrm{Ac}$ and to $66{ }^{\circ} \mathrm{C}$ in Tris buffer indicating that FBP stabilized PKM2 to a similar degree in either solution.

The S437Y mutant of PKM2 which has a sterically occluded FBP binding site $^{7}$, does not bind FBP. Using activity assays it has been shown ${ }^{7}$ that the S437Y mutant of PKM2 is activated by serine but not by FBP. Molecular dynamic simulations indicated that the stability of S437Y PKM2 mutant was significantly decreased compared to the wild type and that the distance between each domain of the mutant was increased ${ }^{24}$. However, no information was found in the literature regarding the oligomeric distribution of the S437Y mutant PKM2 and since the mutant is not activated by FBP, the dimer to tetramer equilibrium should not be affected by addition of FBP It was therefore analyzed in the absence and presence of FBP (Figure 4) as a negative non-FBP binding control. The mass spectrum of the mutant protein showed a different charge state distribution compared to the wild type PKM2. Tetramer, dimer and monomer were all observed. The oligomeric distribution of the mutant is broader than the wild type protein and the reason for this difference is not known but the key observation is that the distribution is not perturbed by the addition of a large molar excess of FBP. The observed oligomeric distribution of the S437Y mutant is therefore consistent with predictions from molecular dynamic simulations ${ }^{24}$. 


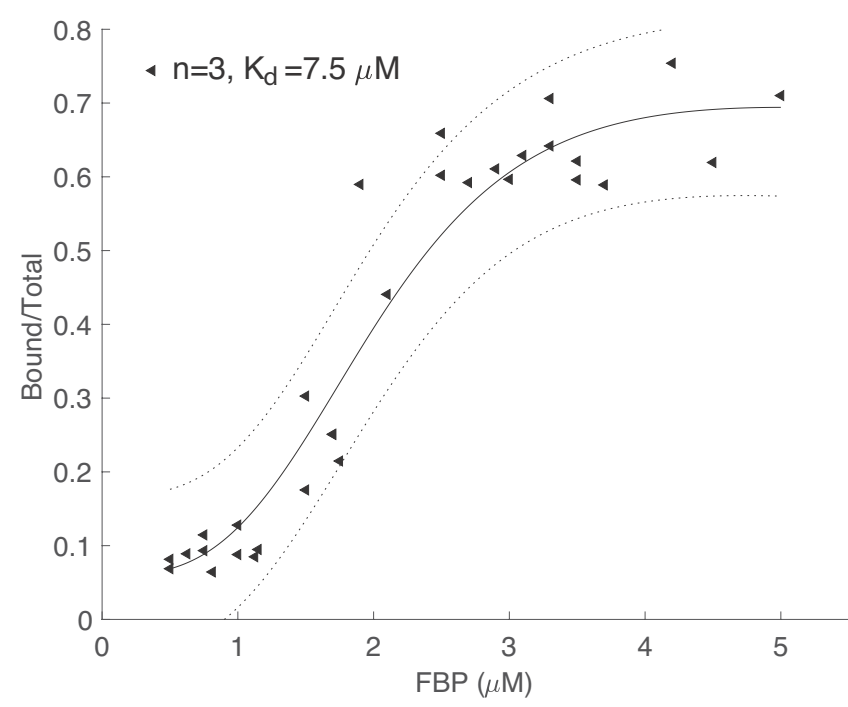

Figure 3: Titration curve for the PKM2-FBP complex. Ion peak area of the FBP-bound PKM2 oligomeric species to total ion peak area (black triangles) measured with MS plotted vs [FBP]. To ensure reproducibility, the data were fitted with the Hill equation and the affinity of the complex was measured to be $7.5 \mu \mathrm{M}$. The binding occurs in a highly positively cooperative manner with a Hill coefficient $n=3$. A $95 \%$ confidence interval is indicated with the dotted lines.

In the presence of $25 \mu \mathrm{M}$ FBP (Figure 4 upper spectrum) no change was observed in the oligomer distribution of the S437Y mutant, and despite the high FBP concentration, no FBP was observed to bind either specifically or nonspecifically to the S437Y mutant of PKM2. Therefore changes to the wild type protein were due to FBP binding.

In this study native MS was applied to analyze co-existing populations of different ligation states of PKM2, a key regulatory node in the glycolytic pathway in tumor cells, thereby generating insights into the allosteric and cooperative mechanism of FBP binding. The various oligomer populations of wild type PKM2 were simultaneously distinguished. It was shown that the oligomeric states of the protein vary with protein concentration and the presence of FBP induces tetramerization of the dimer which is predominantly observed in the absence of added FBP. The overall binding affinity of PKM2 dimers for each other and the average FBP binding affinity to PKM2 were measured. In the presence of FBP fully FBP-bound tetrameric protein (four FBP molecules bound to one tetramer) as well as apo (FBP free) tetramer and dimer were observed. No intermediate ligation states were observed where one, two or three FBP molecules were detected. This suggests that either after binding to apo dimer FBP induces rapid formation and stabilization of the tetramer or FBP can only bind to the tetramer thereby driving the dimer:tetramer equilibrium in the direction of fully FBP bound tetramer. However, it is possible that other ligation states of PKM2 were present but which were not resolved in this experiment. The resolution of the mass spectrometer used for this study would allow the detection of three and possibly two FBP molecules bound if the complex formed was stable within the experimental time frame. However, no intermediate ligation states were observed other than four FBP molecules bound to the tetrameric PKM2.

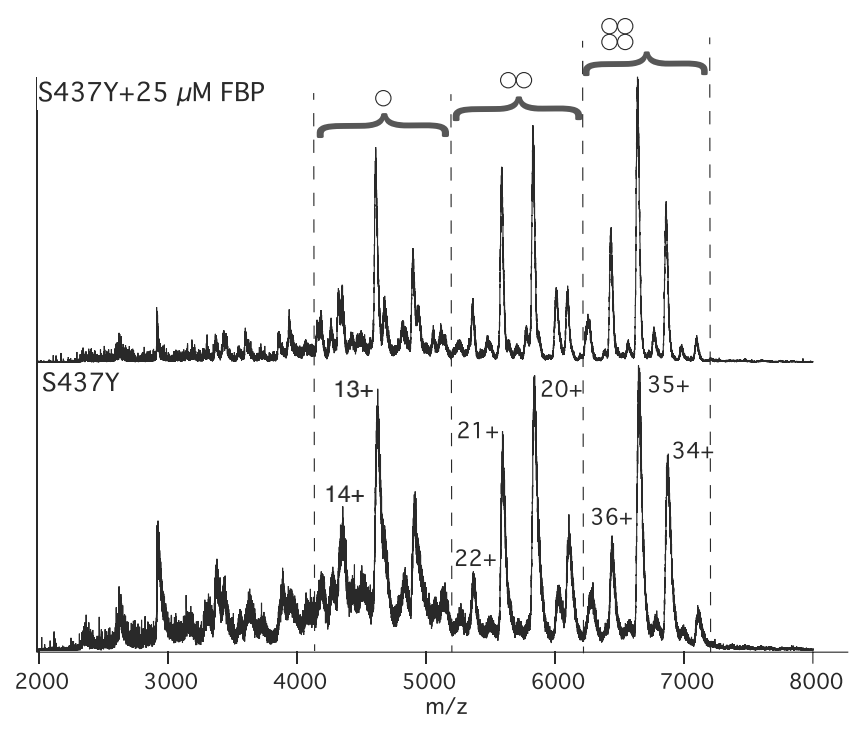

Figure 4: Nano ESI-MS spectra of $2.5 \mu \mathrm{M}$ dimeric S437Y PKM2 mutant in the presence of $25 \mu \mathrm{M}$ FBP (upper spectrum) and in the absence of FBP (lower spectrum). The native state of PKM2 is destabilized by the mutation and tetrameric (four circles), dimeric (two circles), monomeric (one circle) species were observed. The different species are separated with dashed lines. No binding of FBP was observed.

Studying allosteric mechanisms of protein activity is challenging and understanding them is of great biological interest and importance to drug discovery. The high resolution of MS renders it highly applicable to the study of protein ligation states by enabling detection of the mass differences resulting from low mass ligands binding to proteins. This in turn, facilitates the generation of mechanistic insights into ligand binding modes.

The ultimate aim would be to determine a comprehensive model of FBP binding to PKM2 to enable fitting of experimental data to determine $\mathrm{K}_{\mathrm{d}}$ values for the binding of four molecules of FBP to the PKM2 tetramer. This would require additional experiments with higher resolution mass spectrometers $^{25}$ in combination with solution phase determination of affinities and subunit binding equilibria by, for example, ITC and analytical ultracentrifugation ${ }^{26}$ respectively.

\section{ASSOCIATED CONTENT}

\section{Supporting Information}

The Supporting Information is available free of charge on the ACS Publications website. Expression section, ITC and thermal shift assay results and additional mass spectra.

\section{AUTHOR INFORMATION}

\section{Corresponding Author}

* ETH Zurich, Department of Chemistry and Applied Biosciences, CH-8093 Zurich, Switzerland zenobi@org.chem.ethz.ch.

\section{Author Contributions}

All authors have given approval to the final version of the manuscript. 


\section{ACKNOWLEDGMENTS}

We are grateful to Martin Gaugg for valuable help with the Matlab software. We thank the Swiss National Science Foundation (grants no. 200020_140398 and 200020_159929) for financial support of this research.

\section{REFERENCES}

(1) Berg JM, Tymoczko JL, S. L. (2002) Glycolysis and Gluconeogenesis, in Biochemistry. 5th edition. New York: W H Freeman.

(2) Mazurek, S., Boschek, C. B., Hugo, F., and Eigenbrodt, E. (2005)

Pyruvate kinase type M2 and its role in tumor growth and spreading. Semin. Cancer Biol. 15, 300-308.

(3) Mazurek, S. (2011) Pyruvate kinase type M2: a key regulator of the metabolic budget system in tumor cells. Int. J. Biochem. Cell Biol. 43, 969-80.

(4) Gupta, V., Iqbal, mohd A., Kumar, B., and Bamezai, R. N. K. (2015) Tumor Cell Metabolism, in Tumor Cell Metabolism: Pathways, Regulation and Biology, pp 123-142.

(5) Anastasiou, D., Yu, Y., Israelsen, W. J., Jiang, J.-K., Boxer, M. B., Hong, B. S., Tempel, W., Dimov, S., Shen, M., Jha, A., Yang, H., Mattaini, K. R., Metallo, C. M., Fiske, B. P., Courtney, K. D., Malstrom, S., Khan, T. M., Kung, C., Skoumbourdis, A. P., Veith, H., Southall, N., Walsh, M. J., Brimacombe, K. R., Leister, W., Lunt, S. Y., Johnson, Z. R., Yen, K. E., Kunii, K., Davidson, S. M., Christofk, H. R., Austin, C. P., Inglese, J., Harris, M. H., Asara, J. M., Stephanopoulos, G., Salituro, F. G., Jin, S., Dang, L., Auld, D. S., Park, H.-W., Cantley, L. C., Thomas, C. J., and Vander Heiden, M. G. (2012) Pyruvate kinase M2 activators promote tetramer formation and suppress tumorigenesis. Nat. Chem. Biol. $8,839-847$.

(6) Boxer, M. B., Jiang, J., Vander Heiden, M. G., Shen, M., Skoumbourdis, A. P., Southall, N., Veith, H., Leister, W., Austin, C. P., Park, H. W., Inglese, J., Cantley, L. C., Auld, D. S., and Thomas, C. J. (2010) Evaluation of Substituted N, N'-Diarylsulfonamides as Activators of the Tumor Cell Specific M2 Isoform of Pyruvate Kinase. J. Med. Chem. 53, 1048-1055.

(7) Chaneton, B., Hillmann, P., Zheng, L., Martin, A. C. L., Maddocks, O. D. K., Chokkathukalam, A., Coyle, J. E., Jankevics, A., Holding, F. P., Vousden, K. H., Frezza, C., O'Reilly, M., and Gottlieb, E. (2012) Serine is a natural ligand and allosteric activator of pyruvate kinase M2. Nature $491,458-462$.

(8) Yang, J., Liu, H., Liu, X., Gu, C., Luo, R., and Chen, H. F. (2016) Synergistic Allosteric Mechanism of Fructose-1,6-bisphosphate and Serine for Pyruvate Kinase M2 via Dynamics Fluctuation Network Analysis. J. Chem. Inf. Model. 56, 1184-1192.

(9) Christofk, H. R., Vander Heiden, M. G., Harris, M. H., Ramanathan, A., Gerszten, R. E., Wei, R., Fleming, M. D., Schreiber, S. L., and Cantley, L. C. (2008) The M2 splice isoform of pyruvate kinase is important for cancer metabolism and tumour growth. Nature 452, 230 233.

(10) Donovan, K. A., Zhu, S., Liuni, P., Peng, F., Kessans, S. A., Wilson, D. J., and Dobson, R. C. J. (2016) Conformational dynamics and allostery in pyruvate kinase. J. Biol. Chem. 291, 9244-9256.

(11) Dyachenko, A., Gruber, R., Shimon, L., Horovitzb, A., and Sharon, M. (2013) Allosteric mechanisms can be distinguished using structural mass spectrometry. Proc. Natl. Acad. Sci. U. S. A. 110, 7235-7239.

(12) Cubrilovic, D., Haap, W., Barylyuk, K., Ruf, A., Badertscher, M., Gubler, M., Tetaz, T., Joseph, C., Benz, J., and Zenobi, R. (2014) Determination of Protein-Ligand Binding Constants of a Cooperatively Regulated Tetrameric Enzyme Using Electrospray Mass Spectrometry. ACS Chem. Biol. 9, 218-226.

(13) Loo, J. a. (2000) Electrospray ionization mass spectrometry: A technology for studying noncovalent macromolecular complexes. Int. J. Mass Spectrom. 200, 175-186.

(14) Gavriilidou, A. F. M., Gulbakan, B., and Zenobi, R. (2015) Influence of Ammonium Acetate Concentration on Receptor-Ligand Binding Affinities measured by Native nano ESI MS: A Systematic Study. Anal. Chem. 150923215247004

(15) Cubrilovic, D., Barylyuk, K., Hofmann, D., Walczak, M. J., Gräber, M., Berg, T., Wider, G., and Zenobi, R. (2014) Direct monitoring of protein-protein inhibition using nano electrospray ionization mass spectrometry. Chem. Sci. 5, 2794.

5
(16) Loo, J. A. (1997) Studying noncovalent protein complexes by electrospray ionization mass spectrometry. Mass Spectrom. Rev. 16, 1-23. (17) Laszlo, K. J., and Bush, M. F. (2015) Analysis of Native-Like Proteins and Protein Complexes Using Cation to Anion Proton Transfer Reactions (CAPTR). J. Am. Soc. Mass Spectrom. 26, 2152-2161. (18) Belov, M. E., Damoc, E., Denisov, E., Compton, P. D., Horning, S., Makarov, A. A., and Kelleher, N. L. (2013) From protein complexes to subunit backbone fragments: A multi-stage approach to native mass spectrometry. Anal. Chem. 85, 11163-11173.

(19) Morgan, H. P., O’Reilly, F. J., Wear, M. A., O’Neill, J. R., FothergillGilmore, L. A., Hupp, T., and Walkinshaw, M. D. (2013) M2 pyruvate kinase provides a mechanism for nutrient sensing and regulation of cell proliferation. Proc. Natl. Acad. Sci. 110, 5881-5886.

(20) Peschke, M., Verkerk, U. H., and Kebarle, P. (2004) Features of the ESI mechanism that affect the observation of multiply charged noncovalent protein complexes and the determination of the association constant by the titration method. J. Am. Soc. Mass Spectrom. 15, 14241434.

(21) Boeri Erba, E., Barylyuk, K., Yang, Y., and Zenobi, R. (2011) Quantifying protein-protein interactions within noncovalent complexes using electrospray ionization mass spectrometry. Anal. Chem. 83, 92519259.

(22) Root, K., Wittwer, Y., Barylyuk, K., Anders, U., and Zenobi, R. (2017) Insight into Signal Response of Protein Ions in Native ESI-MS from the Analysis of Model Mixtures of Covalently Linked Protein Oligomers. J. Am. Soc. Mass Spectrom. 28, 1863-1875.

(23) Flashner, M., Hollenberg, Paul, L., and Coon, M. J. (1972) Mechanism of Action of Pyruvate Kinase. J. Biol. Chem. 247, 8114-8121. (24) Kalaiarasan, P., Kumar, B., Chopra, R., Gupta, V., Subbarao, N., and Bamezai, R. N. K. (2015) In silico screening, genotyping, molecular dynamics simulation and activity studies of SNPs in Pyruvate Kinase M2. PLoS One 10, 1-21.

(25) Rose, R. J., Damoc, E., Denisov, E., Makarov, A., and Heck, A. J. R. (2012) High-sensitivity Orbitrap mass analysis of intact macromolecular assemblies. Nat. Methods 9, 1084-1086.

(26) Cole, J. L., Lary, J. W., P. Moody, T., and Laue, T. M. (2008) Analytical Ultracentrifugation: Sedimentation Velocity and Sedimentation Equilibrium. Methods Cell Biol. 84, 143-179. 
For the Table of Contents Only

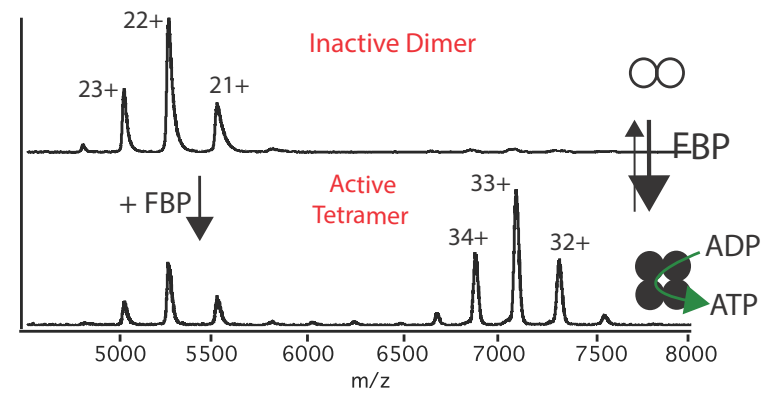

